\title{
Maximum Likelihood Direction of Arrival Estimation using Chicken Swarm Optimization Algorithm
}

\author{
Abhinav Sharma \\ Department of Electrical and Electronics Engineering, \\ University of Petroleum and Energy Studies, Dehradun, Uttarakhand, India. \\ Corresponding author: abhinav.sharma@ddn.upes.ac.in \\ R. Gowri \\ Department of Electrical and Electronics Engineering, \\ University of Petroleum and Energy Studies, Dehradun, Uttarakhand, India. \\ E-mail: rgowri@ddn.upes.ac.in \\ Vinay Chowdary \\ Department of Electrical and Electronics Engineering, \\ University of Petroleum and Energy Studies, Dehradun, Uttarakhand, India. \\ E-mail: vinay.ch1@gmail.com \\ Abhishek Sharma \\ Department of Research \& Development \\ University of Petroleum and Energy Studies, Dehradun, Uttarakhand, India. \\ abhishek_sharma@ddn.upes.ac.in \\ Vibhu Jately \\ MCAST Energy Research Group, \\ Institute of Engineering and Transport, MCAST, Paola, Malta. \\ E-mail: vibhu.jately@mcast.edu.mt
}

(Received September 15, 2020; Accepted December 22, 2020)

\begin{abstract}
Aspects towards the area of array signal processing are majorly confined to two techniques, Direction of arrival (DOA) estimation and adaptive beamforming (ABF). There exist different traditional techniques for estimating the direction of incoming signals such as spectral and Eigen structure-based methods that find the direction of incoming signals. The major drawback of these techniques are that they fail to find the direction of the incoming signal in environments of low signal to noise (SNR). The maximum likelihood (ML) method has an upper hand in terms of statistical performance as compared to conventional methods and finds the direction of signal in low SNR conditions. In this article, the chicken swarm optimization (CSO) algorithm is explored for the optimization of ML function to find the direction of signals in uniform linear arrays (ULA). The algorithm is inspected with respect to the root mean square error (RMSE) and the probability of resolution (PR). Simulation results of the proposed technique prove that the ML-CSO algorithm outperforms other heuristic approaches such as the flower pollination algorithm (FPA) and other conventional techniques such as Capon, multiple signal classification (MUSIC), estimation of signal parameters via rotational invariance technique (ESPRIT) algorithm in lower SNR environment.
\end{abstract}

Keywords- DOA, CSO, FPA, ML, RMSE. 
International Journal of Mathematical, Engineering and Management Sciences

Vol. 6, No. 2, 621-635, 2021

https://doi.org/10.33889/IJMEMS.2021.6.2.038

\section{Introduction}

Active research scope in the area of wireless communication is DOA estimation (Godara, 1997; Stoica and Nehorai, 1990). The process of estimation of the angle of incoming electromagnetic signals to the sensor array is known as DOA estimation, also known as angle of arrival (AOA) estimation. It has found tremendous applications in civilian and military fields such as radar, sonar, tracking of objects, rescue operation, satellite, and wireless communication. Multiple-input and multiple-output (MIMO) arrays (Panzner et al., 2014) and smart antennas are widely used in the $5 \mathrm{G}$ communication system. The key areas of signal processing of MIMO and adaptive antenna (Gross and Volakis, 2005) are DOA estimation and ABF algorithms. Beamforming algorithms performance depends on the efficiency of DOA estimation. Thus, the estimation of direction in the presence of a coherent and multipath channel environment with high accuracy is a crucial requirement of the wireless communication systems at present. There has been significant growth in the development of DOA estimation algorithms over the last decade.

MUSIC (Schmidt, 1986), Bartlett, Capon, Root-MUSIC, ESPRIT (Roy and Kailath, 1989), Pisarenko harmonic decomposition, Matrix pencil are some of the most popular traditional spectral estimation and Eigen structure-based high-resolution DOA estimation algorithm. Sharma and Mathur (2016) compared all the conventional DOA estimation algorithms in an uncorrelated channel environment. All these algorithms fail to find the direction of signals in a low SNR environment. Maximum Likelihood (Stoica and Sharman, 1990) a standard technique is another approach to find the direction of signals. This technique has presented a superior statistical performance in unfavorable conditions such as low SNR and the environment of a coherent channel. Hence, the approach is widely adopted in practical conditions. The ML estimate is computed by maximizing the log-likelihood function which represents that signals from those angles are mostly present in the given samples. The loglikelihood function is highly non-linear and complex, therefore it is difficult to optimize using conventional mathematical approaches. Therefore, metaheuristic (high-level search) approaches have been considered for optimizing the likelihood function for accurate estimation of the direction of signals in a low SNR environment.

Metaheuristic means high-level search; the algorithms find wide applications to solve engineering problems due to their simplicity and flexibility. Over the last two decades research community has developed many new algorithms that are widely used to solve diverse engineering problems. Most of the metaheuristic algorithms are population-based stochastic algorithms which means these algorithms randomly explores the search space in order to avoid local optima and find the global optimum solution. These algorithms are classified into four different classes, viz. evolutionary, swarm, physics, and human-based algorithms. The genetic algorithm is evolutionary-based and simulates the Darwinian evolutionary concept. Karamalis et al. (2001) and Li and Lu (2002) investigated genetic algorithm for ML-DOA estimation in various scenarios of SNR, the number of snapshots, and DOA separation for the linear array. The swarm-based algorithm is inspired by natural colonies, flock, herds, and school and mimics the collective intelligence of agents. Ant colony optimization (ACO), glowworm swarm optimization (GSO), whale optimization algorithm (WOA) are some of the examples of swarm-based algorithms. Sharma and Mathur (2018) explored PSO to estimate the incoming signal direction in the presence of partially correlated, uncorrelated, and coherent channels for linear arrays and compared the results with conventional techniques. 
International Journal of Mathematical, Engineering and Management Sciences

Vol. 6, No. 2, 621-635, 2021

https://doi.org/10.33889/IJMEMS.2021.6.2.038

Errasti-Alcala and Fernandez-Recio (2013) investigated the behavior of five different metaheuristic algorithms viz. particle swarm optimization (PSO), ACO for continuous domains, DE, simulated annealing (SA), GA for finding direction in uniform and non-uniform array. The simulation results show that ACO accurately finds the direction among other metaheuristic algorithms. The physicsbased algorithm is inspired by the physical rules of the universe. Gravitational search algorithm (GSA) is one of the physics-based algorithms and has been used to optimize the ML function to find the direction of uncorrelated signals in linear array (Sharma and Mathur, 2016). Results show that GSA outperforms PSO and other traditional techniques in terms of RMSE and PR. The humanbased algorithm is inspired by human behavior and teaching learning-based algorithms and harmony search algorithms are some of its examples.

In this paper, the author has explored a new metaheuristic algorithm for estimating the direction of the incoming signal for linear arrays. FPA and CSO algorithms are used to optimize the function of ML for finding the direction in an uncorrelated channel scenario. To the best of the author's knowledge, FPA and CSO optimization algorithm are not utilized for DOA estimation in uncorrelated channel environments in linear arrays.

The remainder of the article is structured in the following manner. The data model of ULA for DOA estimation is outlined in section 2. The conventional DOA estimation algorithm is presented in section 3. The mathematical model of FPA and CSO is outlined in sections 4 and 5. Section 6 compares the performance of proposed algorithm with other state of art techniques. The conclusive remarks and scope of future work are presented in section 7.

\section{Data Model}

As shown in Figure 1, consider a ULA having $M$ antenna elements, each being omnidirectional and with uniform spacing $\mathrm{d}$. The sources considered are uncorrelated with relative angle as a broadside to the array axis. The total received signal (D) from the antenna array is mathematically expressed as:

$\bar{y}(t)=\bar{A}(\theta) * \bar{g}(t)+\bar{n}(t)$

where $t$-th represents the time sample, $\bar{x}(t) \bar{x}$ represents the total array output, $\bar{g}(t)$ represents the monochromatic signals vector, $\bar{n}(t)$ represents noise vector, and $\bar{A}(\theta)$ is the array steering matrix with elements of array steering vector. The array steering matrix is therefore defined as given in eq. 2:

$\bar{A}(\theta)=\left[\begin{array}{llll}\bar{a}\left(\theta_{1}\right) & \bar{a}\left(\theta_{2}\right) & \ldots \ldots \ldots & \bar{a}\left(\theta_{D}\right)\end{array}\right]$

where, $\bar{a}(\theta)$ represents the array steering vector, and $\theta$ is the direction of incoming signals. The array steering vector is defined with respect to different array geometries such as linear, circular, planar.

To find the path of incoming signals array correlation matrix is taken into account as it describes the correlation between incoming signals. The array correlation matrix is defined as: 
International Journal of Mathematical, Engineering and Management Sciences

Vol. 6, No. 2, 621-635, 2021

https://doi.org/10.33889/IJMEMS.2021.6.2.038

$\frac{1}{N_{S}} \sum_{k=1}^{N_{S}} \bar{y}(k) \bar{y}^{H}(k)$

where, $\mathrm{N}_{\mathrm{s}}$ represents the number of snapshots, ()$^{\mathrm{H}}$ represents the Hermitian operation respectively.
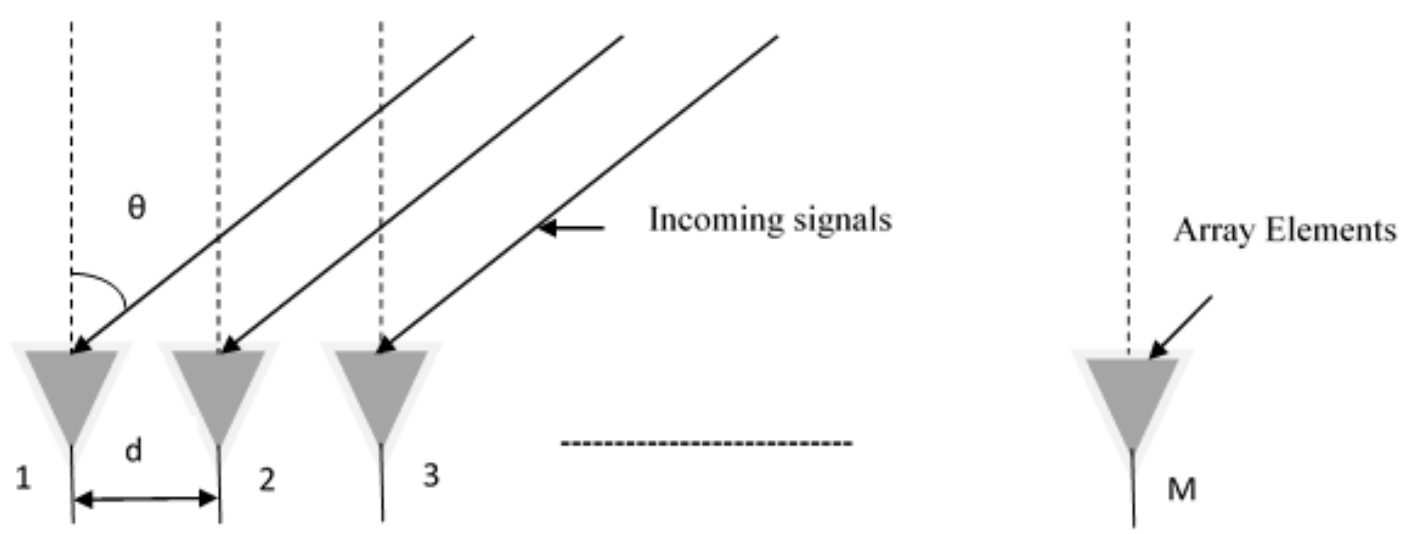

Figure 1. Uniform linear array.

In this article, the authors have assumed the deterministic model for the estimation of the direction of incoming signals. Thus, the signal vector is deterministic with unknown sequences. In this deterministic model, the estimate of the incoming signal's angle, $\theta$, by ML, is acquired by the process of optimization of the non-linear multimodal function, given by:

$f_{D M L}=\operatorname{tr}\left[\left(I_{M}-\bar{A}\left(\bar{A}^{H} \bar{A}\right)^{-1} \bar{A}^{H}\right) \bar{R}\right]$

where, $\operatorname{tr}[]$ is the trace of the bracketed matrix, $\mathrm{I}_{\mathrm{M}}$ representing the identity matrix with the order of $M X M$.

\section{Conventional DOA Estimation Algorithm}

The conventional DOA estimation algorithm is a simple, efficient, less complex algorithm that gives a reasonable performance in a deterministic high SNR environment. These algorithms are categorized as subspace and non-subspace methods. The Non- subspace methods, also known as spectral estimation methods find the incoming signal's direction by detecting the peak of the pseudo spectrum while subspace methods are also known as Eigen structure methods that find incoming signal direction by Eigen decomposition of array correlation matrix into two subspaces of signal and noise. Bartlett, Capon are two of the nonparametric spectral estimation methods whose principle of operation differ how the weights are obtained for building the pseudospectrum. Pisarenko harmonic decomposition, min-norm estimate, MUSIC, root-MUSIC, matrix pencil, and ESPRIT are major Eigen structure methods that exploit the orthogonal signal subspaces for DOA estimation. 
International Journal of Mathematical, Engineering and Management Sciences

Vol. 6, No. 2, 621-635, 2021

https://doi.org/10.33889/IJMEMS.2021.6.2.038

\subsection{CAPON}

CAPON is one of the simple spectral estimation methods which finds the incoming signal direction by maximizing the power contributed by a signal from the desired direction and minimizing the power contributed by interfering signals and noise. The set of array weights obtained by fulfilling this condition is given by:

$\overline{\mathrm{W}}=\frac{\overline{\mathrm{R}}_{\mathrm{XX}}^{-1} \overline{\mathrm{a}}(\theta)}{\overline{\mathrm{a}}^{\mathrm{H}}(\theta) \overline{\mathrm{R}}_{\mathrm{xX}}^{-1} \overline{\mathrm{a}}(\theta)}$

where, $\bar{R}_{x x}^{-1}$ is the array correlation matrix. With this set of array weights, the pseudospectrum is given by:

$\mathrm{P}_{\mathrm{C}}(\theta)=\frac{1}{\overline{\mathrm{a}}^{\mathrm{H}}(\theta) \overline{\mathrm{R}}_{\mathrm{xx}}^{-1} \overline{\mathrm{a}}(\theta)}$

\subsection{MUSIC}

MUSIC is a high-resolution DOA estimation technique proposed by Schmidt in 1986. It is a widely explored technique in the field of wireless communication for estimating the strength, azimuth and elevation angle, and the number of signals in linear, planar, and conformal arrays. The algorithm exploits signal and noise eigen-vector subspace thus also referred to as the subspace method. The algorithm's mathematical model is explained in the steps below:

I. Collect the signals that are incident towards the antenna array and evaluate the array correlation matrix $\bar{R}_{x x}$.

II. Determine the Eigen values and Eigen vectors of the correlation matrix. Eigen vectors analogous to smaller Eigen values represents the noise subspace as:

$\bar{E}_{N}=\left[\begin{array}{lllll}\bar{e}_{1} & \bar{e}_{2} & \ldots \ldots \ldots & \bar{e}_{M-D}\end{array}\right]$

III. At the arrival angle of incoming signals the noise subspace Eigen vectors are orthogonal to the array steering vectors. Therefore, at every arrival angle the Euclidean distance is zero and is given as:

$d^{2}=\bar{a}^{H}(\theta) \bar{E}_{N} \bar{E}_{H}^{H} \bar{a}(\theta)=0$

IV. With the placement of Euclidean distance in the denominator, pseudospectrum can be drawn with the following expression:

$P_{M U S I C}(\theta)=\frac{1}{\left|\bar{a}^{H}(\theta) \bar{E}_{N} \bar{E}_{N}^{H} \bar{a}(\theta)\right|}$

V. Find out the sharp peaks in the pseudospectrum which signifies the arrival angle of the incoming signals. 
International Journal of Mathematical, Engineering and Management Sciences

Vol. 6, No. 2, 621-635, 2021

https://doi.org/10.33889/IJMEMS.2021.6.2.038

\subsection{ESPRIT}

ESPRIT is a robust and computationally efficient DOA estimation algorithm proposed by Roy and Kailath in 1989. The algorithm is initially proposed for frequency estimation and later for estimating the direction of incoming signals in multi-dimensional arrays. It eliminates the search procedure and storage cost ingrained in other DOA estimation algorithm. The fundamental principle of the algorithm is that it utilizes the linear structure of the arrays. The algorithm undertakes that the sensor arrays must organize in matched pairs with fixed displacement vector. The mathematical model of the algorithms is underlined as follows:

I. Collect the signals that are incident towards the antenna array and evaluate the array correlation matrix $\bar{R}_{x x}$.

II. Evaluate the Eigen values and Eigen vectors of the correlation matrix. Signal subspace is represented by the Eigen vectors corresponds to larger Eigen values defined as:

$\bar{E}_{S}=\left[\begin{array}{llllll}\bar{e}_{1} & \bar{e}_{2} & \ldots & \ldots \ldots \ldots & \ldots & \bar{e}_{D}\end{array}\right]$

III. Divide the signal subspace of the two subarrays $\left\{\bar{E}_{1}, \bar{E}_{2}\right\}$ by sorting the elements of the signal subspace.

IV. Determine the matric $\bar{C}$ utilizing the signal subspace $\left\{\bar{E}_{1}, \bar{E}_{2}\right\}$ using the following relation:

$\bar{C}=\left[\begin{array}{l}\bar{E}_{1}^{H} \\ \bar{E}_{2}^{H}\end{array}\right]\left[\begin{array}{ll}\bar{E}_{1} & \bar{E}_{2}\end{array}\right]$

V. Find the Eigen vectors of the matrix $\bar{C}$ and decompose them as:

$\bar{E}_{C}=\left[\begin{array}{ll}\bar{E}_{11} & \bar{E}_{12} \\ \bar{E}_{21} & \bar{E}_{22}\end{array}\right]$

VI. Determine the rotational operator $\bar{\Psi}$ using the relation:

$\bar{\Psi}=-\bar{E}_{12} \bar{E}_{22}^{-1}$

VII. Find the Eigen values of rotational operator.

VIII. Represent the Eigen values in polar form and angle of arrival is estimated using the following relation:

$\theta_{i}=\sin ^{-1}\left(\frac{1}{\beta d} \arg \left(z_{i}\right)\right)$

\section{Flower Pollination Algorithm}

FPA is a stochastic optimization technique proposed by Yang (2012). The algorithm is nature inspired and imitates the process of pollination of flowers. Pollination is a natural evolutionary 
International Journal of Mathematical, Engineering and Management Sciences

Vol. 6, No. 2, 621-635, 2021

https://doi.org/10.33889/IJMEMS.2021.6.2.038

mechanism that generates offspring by moving pollen grains from male anther to female stigma. Pollination can be categorized based on pollination types and pollinators (Abdel-Basset and Shawky, 2019). In the first category, pollination is sub-categorized as self-pollination and crosspollination, as shown in Figure 2. Pollen, from the same plant if is moved from one flower to the other of the same plant then it is self-pollination while if the pollen is moved from one flower to another flower of the different plant then it is cross-pollination. In the second category, pollination is sub-categorized as biotic and abiotic pollination. In biotic pollination, pollen grains are transferred through living creatures on earth like animals and insects while in abiotic pollination pollen grains are transferred through natural resources i.e. wind, water, and gravity. Global pollination is formed by combining cross and biotic-pollination and local-pollination is formed by combining self and abiotic-pollination. The governing principle of FPA is to find the optimum solution by switching between these two pollinations, which is being controlled by switching probability lying in the range [0,1]. FPA finds wide application in optimizing complex multiobjective problems of science and engineering (Alam et al., 2015; Mehta et al., 2020; Pei et al., 2018; Saxena and Kothari, 2016). The mathematical model of the algorithms is underlined as follows:

I. Generate a population of flowers randomly in the search domain.

II. Evaluate the cost of all the flowers and obtain the best solution gbest.

III. Define a switching probability $p$ in the interval $[0,1]$.

IV. Define a random number $r$,

if, $r<p$ opt global pollination defined by

$X_{i}^{t+1}=X_{i}^{t}+L\left(X_{i}^{t}-g_{\text {best }}\right)$

where $X_{i}^{t}$ is the solution vector $X_{i}$ at iteration $\mathrm{t}$ and $\mathrm{L}$ is the Levy flight based step-size defined by,

$L \sim \frac{\lambda \Gamma(\lambda) \sin (\pi \lambda / 2)}{\pi} \frac{1}{s^{1+\lambda}}, \quad\left(s \gg s_{o}>0\right)$

$\Gamma(\lambda)$ is the standard gamma function which is valid for large step-size $s>0$.

else, opt for local pollination defined by,

$X_{i}^{t+1}=X_{i}^{t}+\epsilon\left(X_{j}^{t}-X_{k}^{t}\right)$

where, $X_{j}^{t}, X_{k}^{t}$ are the pollen from various flowers of the same genus of plants, $\epsilon$ is the uniform distribution in the interval $[0,1]$.

V. Evaluate the new solution and update them if better than the old solution.

VI. Keep the current best solution gbest.

VII. Repeat from step 4 in cases where the error is not minimum and the number of iterations has not been reached their maximum value. 


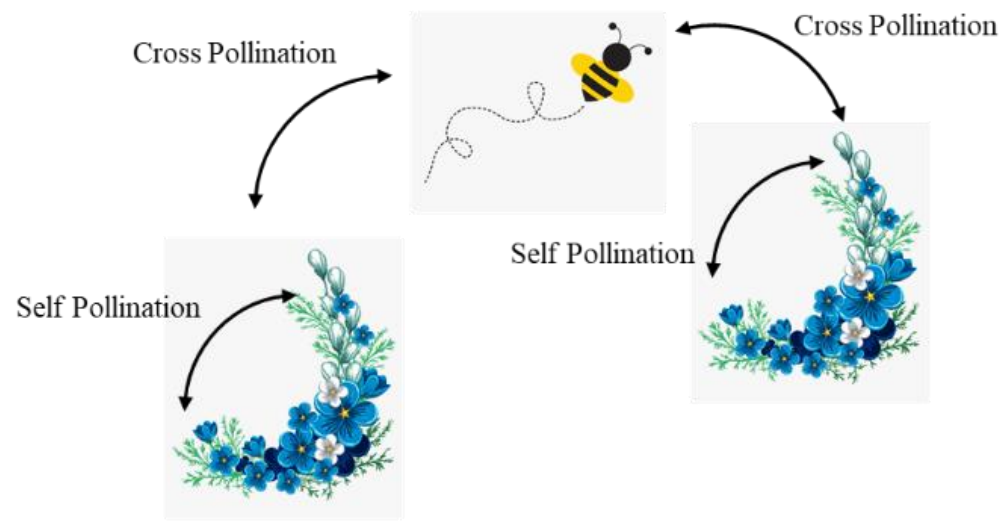

Figure 2. Flower pollination algorithm.

\section{Chicken Swarm Optimization}

CSO is a robust and efficient swarm intelligence technique proposed by Meng et al. (2014). It is inspired by the social behaviour of chickens and imitates the action and hierarchy in the swarm of chicken, which comprises roosters, hens, and chicks, as shown in Figure 3. The algorithm divides chickens into clusters, where each cluster has a dominant rooster, hens, and chicks. The paramount rooster in a congregation dominates the weak chicks. Every cluster of chickens follows the hierarchy, dominance, and mother-child relationship not only to search but also to prevent others from eating their food. Chicken swarm intelligence is extensively used to solve complex optimization problems in the field of science and engineering. Liang et al. (2017) explored CSO for pattern synthesis in the linear and circular antenna array. The algorithm has also been explored in fields such as solar energy (Sharma et al., 2019), wireless sensor networks (WSN) (Shayokh and Shin, 2017; Wang et al., 2017), robotics (Mu et al., 2016), etc.

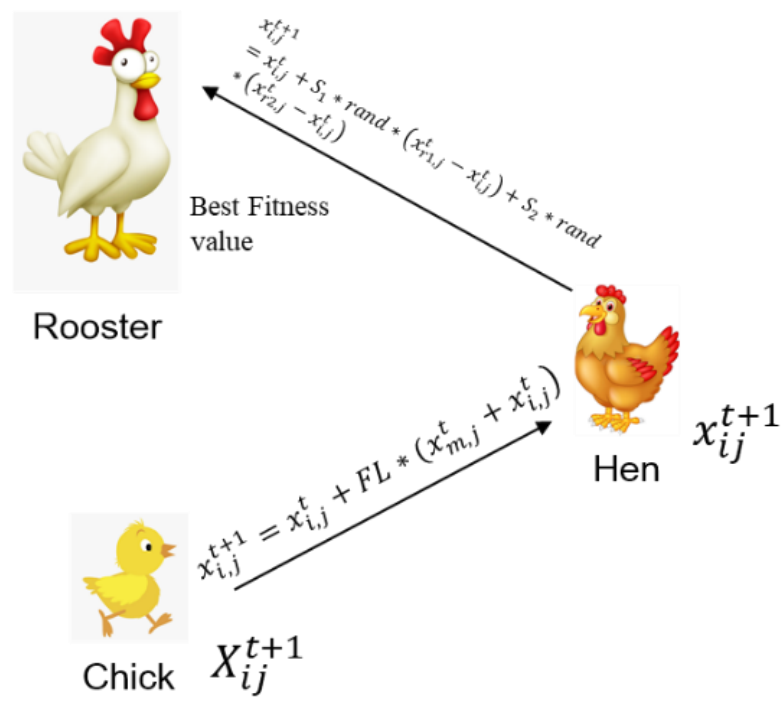

Figure 3. Chicken swarm optimization. 
International Journal of Mathematical, Engineering and Management Sciences

Vol. 6, No. 2, 621-635, 2021

https://doi.org/10.33889/IJMEMS.2021.6.2.038

The mathematical model of the algorithms is underlined as follows:

I. Generate population of chickens randomly in the search domain.

II. Evaluate the cost of all the chickens.

III. Rank each chicken swarm on the basis of cost and organize them in a hierarchal order.

IV. Divide chicken swarm into clusters which includes rooster, hens, and chicks

$\mathrm{V}$. The position of chickens are updated based on the following equations:

if the chicken is a rooster, update the position as:

$x_{i, j}^{t+1}=x_{i, j}^{t} *\left(1+\operatorname{Randn}\left(0, \sigma^{2}\right)\right)$

where,

$$
\sigma^{2}=\left\{\begin{array}{c}
1, \text { if } f_{i} \leq f_{k}, \\
\exp \left(\frac{\left(f_{k}-f_{i}\right)}{\left|f_{i}\right|+\varepsilon}\right), \text { otherwise, } k \in[1, N], k \neq i
\end{array}\right.
$$

if chicken is hen, update the position as:

$x_{i, j}^{t+1}=x_{i, j}^{t}+S 1 * \operatorname{Rand} *\left(x_{r 1, j}^{t}-x_{i, j}^{t}\right)+S 2 * \operatorname{Rand} *\left(x_{r 2, j}^{t}-x_{i, j}^{t}\right)$

where,

$$
S 1=\exp \left(\frac{f_{i}-f_{r 1}}{a b s\left(f_{i}\right)+\varepsilon}\right) \quad \text { and } \quad S 2=\exp \left(\left(f_{r 2}-f_{i}\right)\right)
$$

if the chicken is a chick, update the position as

$x_{i, j}^{t+1}=x_{i, j}^{t}+F L *\left(\mathrm{x}_{\mathrm{m}, \mathrm{j}}^{\mathrm{t}}-\mathrm{x}_{\mathrm{i}, \mathrm{j}}^{\mathrm{t}}\right)$

VI. Estimate the cost of chickens and change their positions if the new cost is better than the previous one.

VII. Repeat from step 3 in cases where the error is not minimum and a number of iterations have not been reached their maximum value.

\section{Implementation of CSO and FPA for DOA Estimation}

I. Generate the search agents of the second-order dimension representing the direction of incoming signals in the range $\left[-90^{\circ}\right.$ to $\left.90^{\circ}\right]$.

II. Evaluate the cost of all search agents using deterministic ML cost function given in eq. (4).

III. Update the position of all search agents using CSO and FPA.

IV. The positions corresponding to minimum cost represents the angle of incoming signals.

\section{Results and Discussion}

Simulation results of DOA estimation of Capon, MUSIC, ESPRIT, ML-FPA, and ML-CSO algorithm using MATLAB software are presented and discussed in this section. The two narrowband plane waves are directed towards 10 element ULA from $25^{\circ}$ and $28^{\circ}$ with 100 number of snapshots. The signals with narrow angular of $3^{0}$ are considered in order to examine the 
International Journal of Mathematical, Engineering and Management Sciences

Vol. 6, No. 2, 621-635, 2021

https://doi.org/10.33889/IJMEMS.2021.6.2.038

robustness of the algorithm. Simulation of the uncorrelated deterministic BPSK signals is performed. Variation of SNR having a step size of $2 \mathrm{~dB}$ is performed from $-20 \mathrm{~dB}$ to $30 \mathrm{~dB}$. In CAPON and MUSIC algorithm the search range is $\left[-90^{\circ}, 90^{\circ}\right]$ where the peaks of the pseudospectrum are identified. In ESPRIT algorithm the direction of incoming signals is obtained using the total least square (TLS) criterion. In all the simulation, 100 Monte-Carlo trials have been considered. Table 1 summarizes the parameters considered for ML-FPA and ML-CSO algorithm.

Table 1. Parameters of CSO and FPA.

\begin{tabular}{|c|c|c|}
\hline & $\begin{array}{c}\text { Population Size } \\
\text { (Popsize) }\end{array}$ & Parameters \\
\hline CSO & 100 & $\mathrm{RS}=0.15 *$ Popsize, $\mathrm{HN}=0.7 *$ Popsize, $\mathrm{CH}=0.15 *$ Popsize, $\mathrm{MHN}=0.5 * \mathrm{HN}$, FL $\in[0.5,0.9]$ \\
\hline FPA & 100 & Probability Switch=0.8, Scale factor=0.01, Levy distribution index $=1.5$ \\
\hline
\end{tabular}

The author has contrasted the efficiency of five different algorithms with respect to RMSE, PR, and later analyzed the rate of convergence and distribution spread of ML-FPA and ML-CSO algorithm.

\section{(a) Root Mean Square Error (RMSE)}

RMSE is the error obtained by subtracting true direction of the signal and estimated direction of signals and is mathematically calculated as:

$R M S E=\sqrt{\frac{1}{N_{n} N_{\text {runs }}} \sum_{l=1}^{N_{\text {runs }}} \sum_{i=1}^{N_{n}}\left[\hat{\theta}_{i}(l)-\theta_{i}\right]^{2}}$

where, $\mathrm{N}_{\text {runs }}$ and $\mathrm{N}_{\mathrm{n}}$ are the independent Monte-Carlo trials, $\hat{\theta}_{i}(l)$ is the evaluated angle of the $\mathrm{i}^{\text {th }}$ direction of signal in the $\mathrm{l}^{\text {th }}$ run, $\theta_{i}$ is the true direction of the $\mathrm{i}^{\text {th }}$ source.

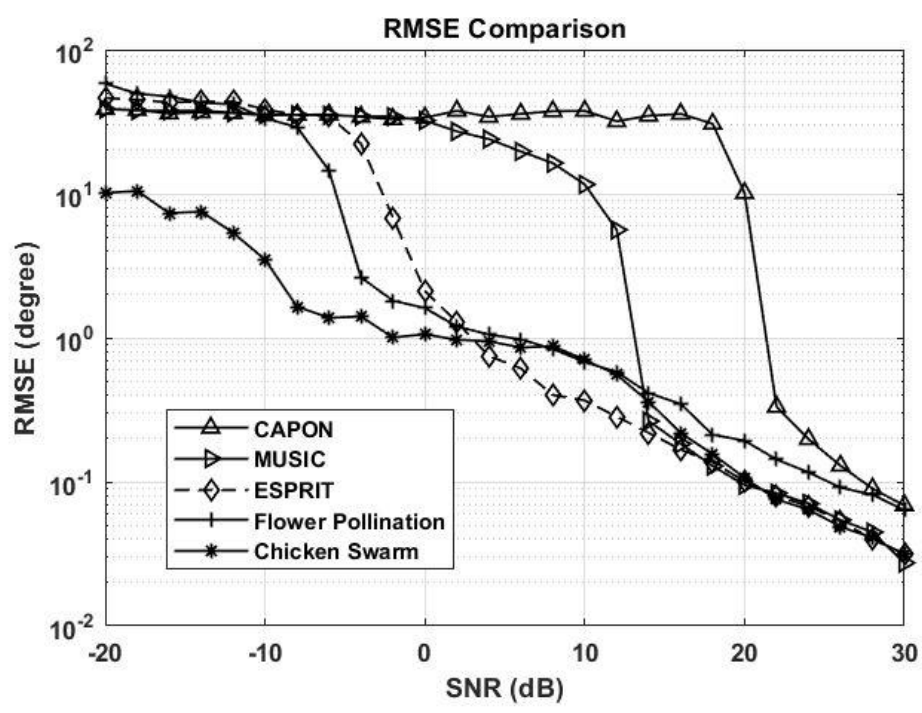

Figure 4. Plot of RMSE versus SNR for Capon, MUSIC, ESPRIT, FPA, CSO. 
International Journal of Mathematical, Engineering and Management Sciences

Vol. 6, No. 2, 621-635, 2021

https://doi.org/10.33889/IJMEMS.2021.6.2.038

Figure 4 depicts the plot of RMSE of conventional techniques Capon, MUSIC, ESPRIT, and the ML-FPA and ML-CSO versus SNR. The simulation result shows that the CSO technique outperforms conventional techniques and FPA in low SNR conditions. In high SNR conditions above $15 \mathrm{~dB}$ MUSIC, ESPRIT, and CSO algorithm performs equally. This proves that conventional techniques MUSIC, ESPRIT algorithm can be utilized for DOA estimation for deterministic signals.

\section{(b) Probability of Resolution (PR)}

This is the algorithm's ability to resolve signals that are closely spaced. PR examines the robustness of the algorithm. If the two sources are arriving at an angle $\theta_{1}, \theta_{2}$ and if the difference of estimated and actual angle of both the sources is less than half of the difference between actual angles, then the sources are said to be resolved. The resolution probability versus SNR for Capon, MUSIC, ESPRIT, ML-FPA, and ML-CSO algorithm is depicted in Figure 5. The simulation result shows that in lower SNR conditions, less than $0 \mathrm{~dB}$ the CSO shows good resolution to resolve closely spaced signals as compared to other algorithms. Although in high SNR conditions, above $12 \mathrm{~dB}$ MUSIC, ESPRIT and FPA presents 100 percent probability and at $22 \mathrm{~dB}$ Capon shows 100 percent probability. Figure 6 shows the resolution probability in terms of varying angular separation of $3^{0}$, $2.5^{0}, 2^{0}, 1.5^{0}, 1^{0}, 0.5^{0}$ for various algorithms. The SNR of all individual algorithm was fixed at which they have obtained the resolution probability of 1 at $3^{0}$ separation angle. The presented result depicts that Capon, MUSIC, ESPRIT, ML-FPA, ML-CSO algorithm attains resolution probability of 1 at $22 \mathrm{~dB}, 16 \mathrm{~dB}, 10 \mathrm{~dB}, 18 \mathrm{~dB}, 14 \mathrm{~dB}$. ML-CSO algorithm precisely estimates the angle of incoming signals with a separation angle of $0.5^{0}, 1^{0}$ at an SNR of $14 \mathrm{~dB}$.

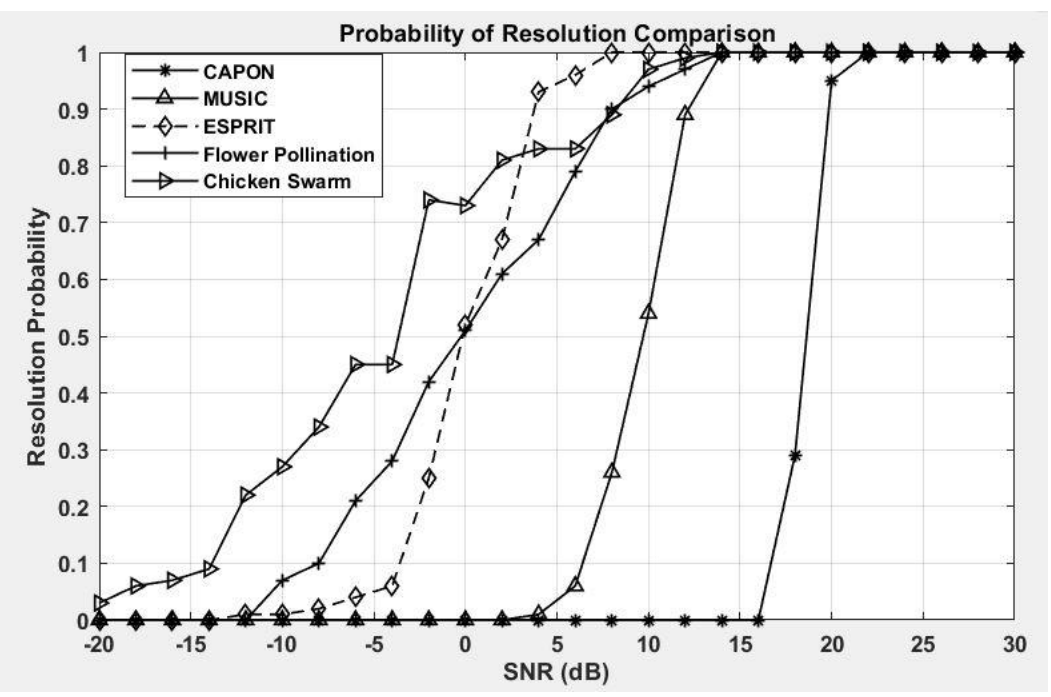

Figure 5. Plot of Resolution Probability versus SNR values for Capon, MUSIC, ESPRIT, FPA, CSO. 
International Journal of Mathematical, Engineering and Management Sciences

Vol. 6, No. 2, 621-635, 2021

https://doi.org/10.33889/IJMEMS.2021.6.2.038

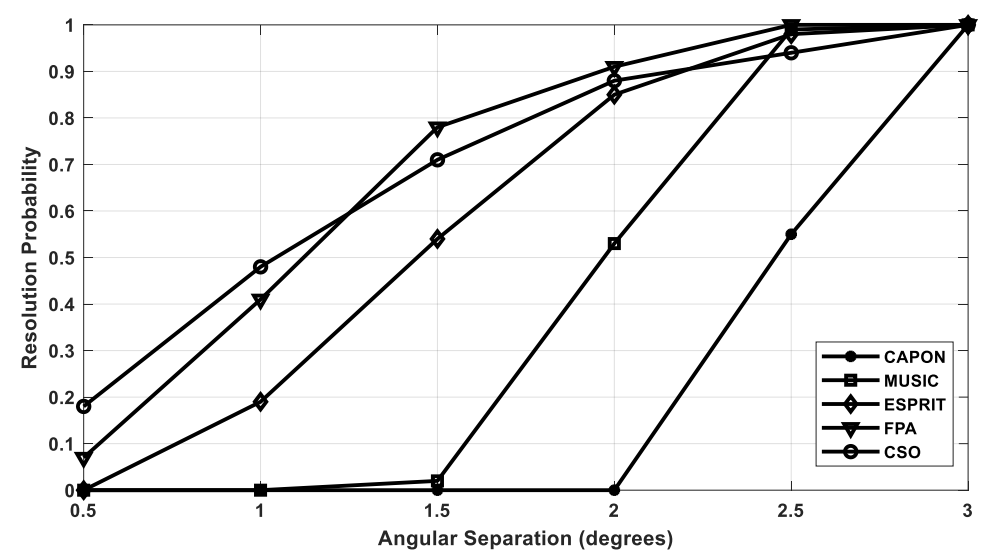

Figure 6. Plot of Resolution Probability versus SNR values for Capon, MUSIC, ESPRIT, FPA, CSO.

\section{(c) Convergence Plot and Box Plot}

The convergence plot is a dominant parameter for analyzing the performance of the optimization algorithm. The convergence plot of RMSE for ML-CSO and ML-FPA is depicted in Figure 7 in which the algorithm will terminate in 1000 iterations. The simulation results show that the MLCSO algorithm converges much earlier than ML-FPA to find the direction of deterministic signals. The distribution of RMSE in terms of boxplot for ML-FPA and ML-CSO algorithm with 100 Monte-Carlo runs is shown in Figure 8. The results depict that the ML-CSO algorithm outperforms the ML-FPA algorithm in terms of accuracy and reliability.

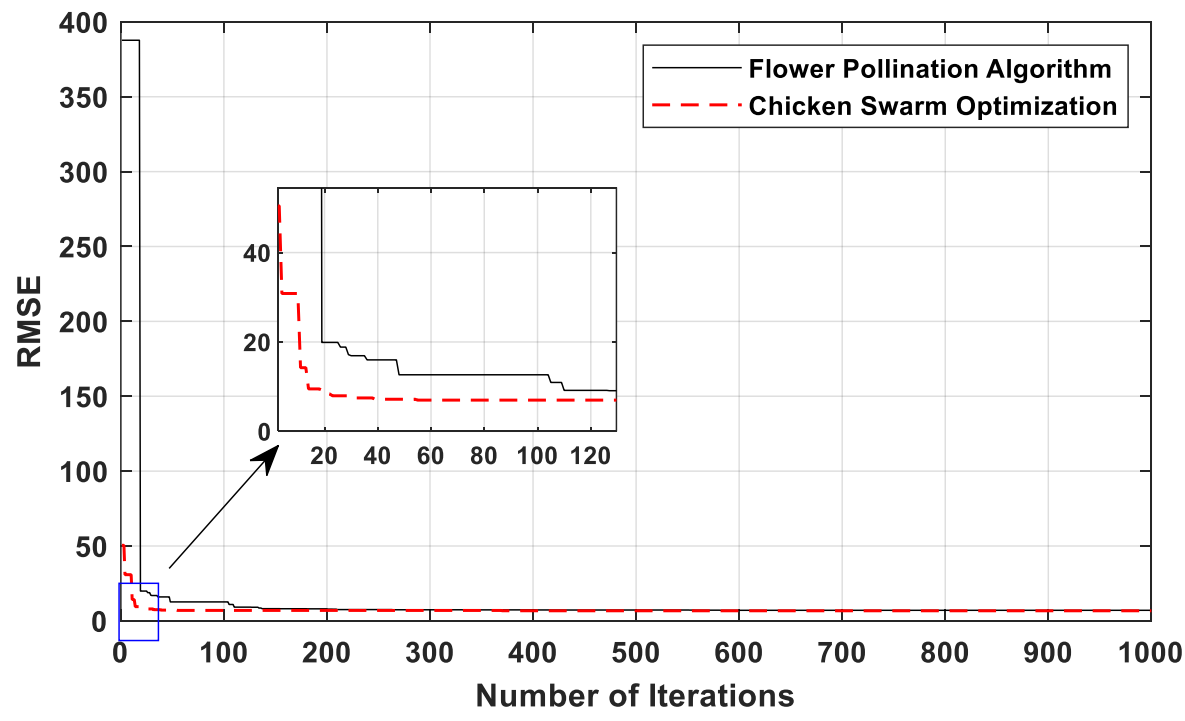

Figure 7. RMSE Convergence plot of FPA and CSO algorithm 
International Journal of Mathematical, Engineering and Management Sciences

Vol. 6, No. 2, 621-635, 2021

https://doi.org/10.33889/IJMEMS.2021.6.2.038

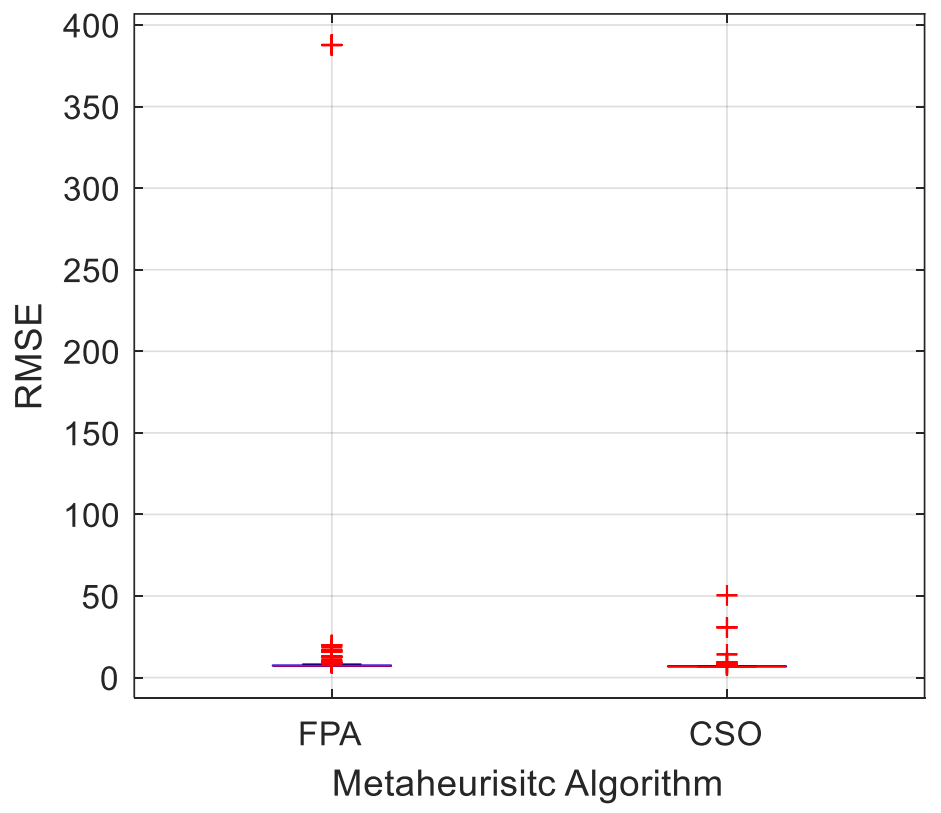

Figure 8. Boxplot of FPA and CSO algorithm

\section{Conclusion}

In this article, CSO is explored for finding the direction of closely spaced signals which are directed towards the linear array. The direction of signals was found by optimizing deterministic ML function. RMSE and PR were the two parameters that have been considered to test the efficiency of the proposed algorithm. The main findings of the article are:

- ML-CSO algorithm shows robust performance and accurately finds the direction of the incoming signal in a low SNR environment.

- ML-CSO algorithm shows the best resolution probability and accurately finds the direction of closely separated signals.

- ML-CSO algorithm converges faster than ML-FPA and presents reliable results.

The future prospects of this work are to analyze the performance of the algorithm for different array configurations such as a circular array, planar array, and cylindrical array. The robustness of the algorithm can be analyzed by exploring the algorithm in a multipath and coherent channel environment. With the widespread growth in digital circuitry and embedded system, using fieldprogrammable gate arrays (FPGAs) the real-time implementation of DOA estimation algorithms can be done.

\section{Conflict of Interest}

The authors have no conflict of interest to publish this paper. 
International Journal of Mathematical, Engineering and Management Sciences

Vol. 6, No. 2, 621-635, 2021

https://doi.org/10.33889/IJMEMS.2021.6.2.038

\section{Acknowledgements}

The authors would like to express their sincere thanks to the editor and anonymous reviewers for their time and valuable suggestions that lead to substantial improvement in the manuscript.

\section{References}

Abdel-Basset, M., \& Shawky, L.A. (2019). Flower pollination algorithm: a comprehensive review. Artificial Intelligence Review, 52(4), 2533-2557.

Al Shayokh, M., \& Shin, S.Y. (2017). Bio inspired distributed WSN localization based on chicken swarm optimization. Wireless Personal Communications, 97(4), 5691-5706.

Alam, D.F., Yousri, D.A., \& Eteiba, M.B. (2015). Flower pollination algorithm based solar PV parameter estimation. Energy Conversion and Management, 101 , 410-422.

Errasti-Alcala, B., \& Fernandez-Recio, R. (2013). Performance analysis of metaheuristic approaches for single-snapshot DOA estimation. IEEE Antennas and Wireless Propagation Letters, 12, 166-169.

Godara, L.C. (1997). Application of antenna arrays to mobile communications. II. Beam-forming and direction-of-arrival considerations. Proceedings of the IEEE, 85(8), 1195-1245.

Gross, F.B. (2005). Smart Antennas for Wireless Communications with MATLAB. McGraw-Hill.

Karamalis, P., Marousis, A., Kanatas, A., \& Constantinou, P. (2001, May). Direction of arrival estimation using genetic algorithms. In IEEE VTS 53rd Vehicular Technology Conference, Spring 2001. Proceedings (Cat. No. 01CH37202) (Vol. 1, pp. 162-166). IEEE. Rhodes, Greece.

Li, M., \& Lu, Y. (2002). Genetic algorithm based maximum likelihood DOA estimation. International Radar Conference, (pp. 502-506).

Liang, S., Feng, T., \& Sun, G. (2017). Sidelobe-level suppression for linear and circular antenna arrays via the cuckoo search-chicken swarm optimisation algorithm. IET Microwaves, Antennas \& Propagation, 11(2), 209-218.

Mehta, I., Singh, G., Gigras, Y., Dhull, A., \& Rastogi, P. (2020). Robotic Path Planning Using Flower Pollination Algorithm. Recent Advances in Computer Science and Communications (Formerly: Recent Patents on Computer Science), 13(2), 191-199.

Meng, X., Liu, Y., Gao, X., \& Zhang, H. (2014, October). A new bio-inspired algorithm: chicken swarm optimization. In International conference in swarm intelligence (pp. 86-94). Springer, Cham.

Mu, Y., Zhang, L., Chen, X., \& Gao, X. (2016, August). Optimal trajectory planning for robotic manipulators using chicken swarm optimization. In 2016 8th International Conference on Intelligent Human-Machine Systems and Cybernetics (IHMSC) (Vol. 2, pp. 369-373). IEEE. Hangzhou, China.

Panzner, B., Zirwas, W., Dierks, S., Lauridsen, M., Mogensen, P., Pajukoski, K., \& Miao, D. (2014, December). Deployment and implementation strategies for massive MIMO in 5G. In 2014 IEEE Globecom Workshops (GC Wkshps) (pp. 346-351). IEEE. Austin, TX, USA.

Pei, T., Hao, X., \& Gu, Q. (2018). A novel global maximum power point tracking strategy based on modified flower pollination algorithm for photovoltaic systems under non-uniform irradiation and temperature conditions. Energies, 11(10), 2708.

Roy, R., \& Kailath, T. (1989). ESPRIT-estimation of signal parameters via rotational invariance techniques. IEEE Transactions on acoustics, speech, and signal processing, 37(7), 984-995.

Saxena, P., \& Kothari, A. (2016). Linear antenna array optimization using flower pollination algorithm. SpringerPlus, 5(1), 306. 
International Journal of Mathematical, Engineering and Management Sciences

Vol. 6, No. 2, 621-635, 2021

https://doi.org/10.33889/IJMEMS.2021.6.2.038

Schmidt, R. (1986). Multiple emitter location and signal parameter estimation. IEEE Transactions on Antennas and Propagation, 34(3), 276-280.

Sharma, A., \& Mathur, S. (2016). Performance analysis of adaptive array signal processing algorithms. IETE Technical Review, 33(5), 472-491.

Sharma, A., \& Mathur, S. (2016, March). Deterministic maximum likelihood direction of arrival estimation using GSA. In 2016 International Conference on Electrical, Electronics, and Optimization Techniques (ICEEOT) (pp. 415-419). IEEE.

Sharma, A., \& Mathur, S. (2018). Comparative analysis of ML-PSO DOA estimation with conventional techniques in varied multipath channel environment. Wireless Personal Communications, 100(3), 803817.

Sharma, A., Pachauri, R., Sharma, A., \& Raj, N. (2019, November). Extraction of the solar PV module parameters using chicken swarm optimization technique. In 2019 Women Institute of Technology Conference on Electrical and Computer Engineering (WITCON ECE) (pp. 45-48). IEEE.

Stoica, P., \& Nehorai, A. (1990). Performance study of conditional and unconditional direction-of-arrival estimation. IEEE Transactions on Acoustics, Speech, and Signal Processing, 38(10), 1783-1795.

Stoica, P., \& Sharman, K.C. (1990). Maximum likelihood methods for direction-of-arrival estimation. IEEE Transactions on Acoustics, Speech, and Signal Processing, 38(7), 1132-1143.

Wang, Q., \& Zhu, L. (2017, May). Optimization of wireless sensor networks based on chicken swarm optimization algorithm. In: AIP conference proceedings (Vol. 1839, No. 1, p. 020197). AIP Publishing LLC.

Yang, X.S. (2012, September). Flower pollination algorithm for global optimization. In: International conference on unconventional computing and natural computation (pp. 240-249). Springer, Berlin, Heidelberg. 